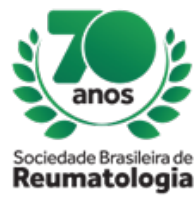

\title{
PANSINUSITE AND BILATERAL CHRONIC OTOMASTOIDITIS IN PATIENT WITH GRANULOMATOSIS WITH POLYANGIITIS (GPA)
}

Gabriella Stefenoni Kruger (HC UFTM, Uberaba, MG, Brasil), Sérgio Mauro Resende Moura (HC UFTM, Uberaba, MG, Brasil), alfredo julio gonzaga horbylon (HC-UFTM, uberaba, MG, Brasil), Maíra Rodrigues Pereira (HC-UFTM , Uberaba , MG, Brasil), Reginaldo Botelho Teodoro (HC-UFTM , Uberaba, MG, Brasil)

\section{BACKGROUND}

Granulomatosis with Polyangiitis (GPA) is a rare autoimmune disease that presents with vasculitis and granulomatosis of small and medium blood vessels.It has a higher prevalence in Caucasians between 6574 years, but can occur at any age.It mainly affects the upper airways, lung parenchyma,kidneys,skin and nervous system.About $80-95 \%$ of patients have antineutrophil cytoplasmic antibodies (ANCA).

\section{CASE REPORT}

J.C.S, 38 years, male,presented with pansinusitis and bilateral chronic otomastoiditis. The diagnostic hypothesis of GPA was confirmed with c-ANCA reagent and biopsy of the nasal septum and compatible skin. The patient presented prolonged hospitalization, from 19 March 2019 until 30 May 2019.During hospitalization it was necessary to use several antibiotics (ceftriaxone,cefepime,clindamycin, imipenem,vancomycin)due to recurrent infection. The patient also presented with acute renal insufficiency with proteinuria during hospitalization.A renal biopsy was performed, which evidenced pauci-immune necrotizing segmental glomerulonephritis, which associated with the clinical data was defined as Granulomatous Polyangiitis (GPA).Patient also presented with polyuria with a diagnostic hypothesis by the team of nephrogenic diabetes insipidus due to prolonged use of nephrotoxic antibiotics. At the beginning of the hospital stay, the patient presented lesions caused by the disease, hemorrhagic pustules of large extension in the distal regions of the upper and lower limbs and erythrematous nodules (Figures 1 and 2), which are in improvement, nevertheless presents a sequela (necrosis in mummification process in distal region of right 2 nd finger). The patient also presented critical patient polyneuropathy, Marasmo-Kwashiokor malnutrition, urinary incontinence and enteral nutrition. For underlying disease (Granulomatosis with Polyangiitis), during hospitalization, pulse therapy with methylprednisolone and cyclophosphamide was performed. Patient was discharged on 30 May 2019, in good condition, remaining afebrile for 6 days and stable hemodynamically. After discharge the patient will maintain multiprofessional outpatient follow-up.

\section{CONCLUSION}

Nasal, sinus, oral, laryngopharyngeal, tracheal and ear signs and symptoms are observed in more than $90 \%$ of GPA cases. In GPA, sinus symptoms include pain, persistent bloody or purulent rhinorrhea congestion, recurrent epistaxis and nasal crusts. Inflammation can lead to erosion of the mucosa membrane as well as destruction of the underlying bone and cartilage, resulting in septum perforation, and in many cases, nasal septum collapse or "saddle" deformity of the nose, favoring infectious processes. Conductive, sensorineural or mixed hearing loss may develop in GPA. Almost $80 \%$ of patients with GPA present with renal disease, with progressive glomerulonephritis being the most serious complication. Approximately $50 \%$ of the patients present cutaneous lesions such as palpable purpura, digital gangrene, erythrematosus nodules, skin ulcers, subungual hemorrhage, among others. 